\title{
Design of a Resonant-Cavity-Enhanced Photodetector for High-Speed Applications
}

\author{
Hsin-Han Tung and Chien-Ping Lee, Senior Member, IEEE
}

\begin{abstract}
We present a theoretical study of the effects of light field distribution on the frequency response of a resonant-cavityenhanced p-i-n photodetector. Taking advantage of the flexibility of cavity design, one can tailor the light field distribution in the absorption region. Because of the difference in velocities of the carriers, the speed performances of the detector depend on the field distribution and the cavity design. The results of our work indicate that when the maximum of light field intensity happens near the $p^{+}$edge of the depletion layer, the device shows the best speed performance. The frequency response, the impulse response, and the step response have been calculated for different structures to demonstrate the importance of the field distribution.
\end{abstract}

Index Terms-Frequency response, impulse response, quantum efficiency, resonant-cavity-enhanced photodector, step response.

\section{INTRODUCTION}

$\mathbf{H}$ IGH-SPEED and high-sensitivity photodetectors are important for high-bit-rate optical communication systems [1], [2]. The detectors are required to have a wide bandwidth and a high quantum efficiency. For conventional p-i$\mathrm{n}$ photodiodes, the quantum efficiency $\eta$ is limited by the absorption coefficient $\alpha$ and the absorbing layer thickness $d$. The highest $\eta$ obtainable for detectors with a perfect antireflecting coating is $\eta=1-\exp (-\alpha d)$. To achieve high $\eta$, a thick absorbing layer is required. However, a thick absorbing layer means a long carrier transit time which reduces the device's speed. Resonant-cavity-enhanced (RCE) photodetectors provide a solution to this problem [3], [4]. For such detectors, a thin absorbing layer is put inside a Fabry-Perot cavity. The feedback mirrors of the Fabry-Perot cavity are usually comprised of quarter wavelength stacks (QWS's) [5] with a periodic modulation of the refractive index. At resonance, construction interference is built up within the cavity to enhance the internal optical field intensity. Since the generation rate, $g$, of the electron-hole pairs is proportional to the light intensity $J_{p h}$, i.e., $g=\alpha J_{p h}, g$ can be greatly enhanced. So only a very thin absorbing layer is needed to achieve high quantum efficiency. RCE photodetectors with nearly $100 \%$ quantum efficiency [6], [7] have been demonstrated.

Manuscript received July 8, 1996; revised January 22, 1997. This work was supported by the National Science Council of the Republic of China under Contract NSC84-2215-E009-039.

H.-H. Tung is with the Department of Electrical Engineering, National LienHo Junior College of Technology and Commerce, Miao Li, Taiwan, R.O.C.

C.-P. Lee is with the Department of Electronics Engineering, National Chiao Tung University, Hsin Chu, Taiwan, R.O.C.

Publisher Item Identifier S 0018-9197(97)03065-0.
The speed of a photodetector is primarily determined by the combined effect of the capacitance of the detector and the transit time for the photocarriers to move through the absorbing layer. For a high-speed detector, the transit time has to be very short. When the area of the detectors is made sufficiently small, the influence from the capacitance is reduced and the effect of the transit time dominates. Wey et al. have demonstrated a $2 \mu \mathrm{m} \times 2 \mu \mathrm{m}$ GaInAs-InP p-i-n photodiode with a 3-dB bandwidth as high as $110 \mathrm{GHz}$ [8], [9]. Bowers and Burrus have indicated that bandwidths in excess of $200 \mathrm{GHz}$ should be possible [10].

For high-speed operation, the electric field throughout the intrinsic layer should be very high so that the carriers travel at the saturation velocity during most of the transit. Usually the electron saturation velocity is greater than the hole saturation velocity; taking advantage of this property, one can improve speed performance for proper device designs. On the other hand, the distributions of photogenerated carriers can also have significant influence on the speed response because the average distance that carriers travel depends on the distribution. In this paper, the performances of RCE photodetectors with different carrier profiles as a result of different cavity design are compared. We found that by proper design, we can tailor the carrier profile in the absorbing region to maximize the speed of the detector.

\section{RCE p-i-n PHOTODIODE}

Fig. 1 shows the schematic diagram of a RCE p-i-n photodiode used in this study. The structure is similar to that designed by Tang et al. [7]. The thickness of the $\mathrm{p}^{+}$, i, $\mathrm{n}^{+}$layers are denoted as $L_{p}, L_{i}$, and $L_{n}$, respectively. The front mirror is composed of a half- $\lambda$-thick $\mathrm{SiO}_{2}$ plus one $\mathrm{Si}-\mathrm{SiO}_{2}$ quarter-wave pair, and the back mirror consists of a $0.5 \lambda$-thick GaAs layer and a 27-period GaAs-AlAS QWS, where $\lambda$ is the designed wavelength, $1.3 \mu \mathrm{m}$, divided by the refractive index of the material. The absorption coefficient of the intrinsic InGaAs layer is assumed to be $1.16 \times 10^{4} \mathrm{~cm}^{-1}$ at $1.3 \mu \mathrm{m}$. The quantum efficiency of the resonant detector can be calculated numerically by using the transfer-matrix method (TMM) [11] and is equal to $1-R-T$, where $R$ is the power reflectivity and $T$ is the power transmission. Fig. 2 shows the calculated quantum efficiency versus wavelength for $L_{p}=$ $3 / 4 \mu \mathrm{m}, L_{i}=1 / 4 \mu \mathrm{m}$, and $L_{n}=5 / 4 \mu \mathrm{m}$. The dashed curve is for the reference photodiode without a resonant cavity. It is noticed that the quantum efficiency is significantly increased by a factor of 10 (from $10 \%$ to nearly $100 \%$ ) at $1.3 \mu \mathrm{m}$. 


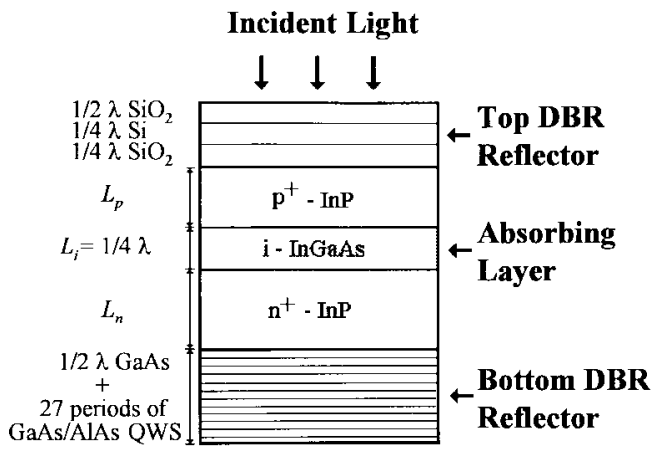

Fig. 1. Schematic representation of the studied RCE p-i-n photodetector. Here $\lambda$ is the design wavelength, $1.3 \mu \mathrm{m}$, divided by the refractive index of each layer.

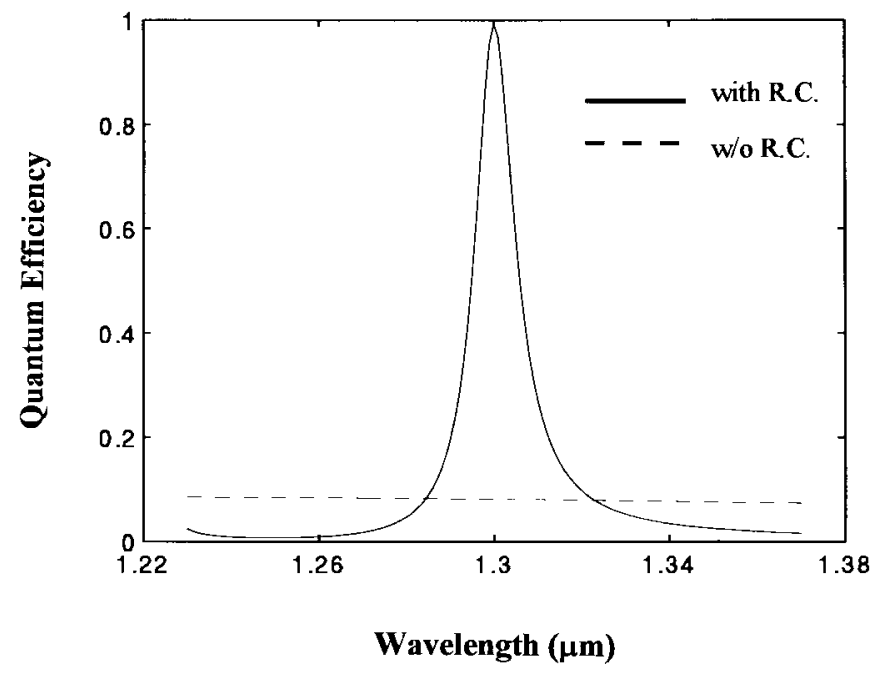

Fig. 2. Calculated quantum efficiency for the studied RCE photodiode with $L_{p}=3 / 4 \lambda, L_{i}=1 / 4 \lambda$, and $L_{n}=5 / 4 \lambda$ (solid line). The diode is designed at $1.3-\mu \mathrm{m}$ wavelength. Quantum efficiency for conventional photodiode without resonant cavity is also shown for comparison (dashed line).

The quantum efficiency of the resonant detector can be also obtained approximately as [12] (1), shown at the bottom of the page, where $R_{1}$ and $R_{2}$ are reflectivities of the top and bottom mirrors, respectively, $\theta_{k}$ is the round-trip phase delay of the resonant mode within the cavity, and $k$ is the resonant mode. The effective absorption coefficient, $\alpha_{\mathrm{eff}}=\alpha S$, and the factor $S$ is between 0 and 2 depending on the standing wave effect. From [12], we know that when the condition

$$
R_{1}=R_{2} \exp \left(-2 \alpha_{\mathrm{eff}} L_{i}\right)
$$

is satisfied, the quantum efficiency at a certain $\alpha_{\text {eff }} L_{i}$ value is maximized. The parameters we use in the present calculation at $\lambda=1.3 \mu \mathrm{m}$ yield $\theta_{k} \cong 2 k \pi(k=4), S \cong 1, R_{1} \cong$ $0.8, R_{2} \cong 1$ and $\alpha_{\text {eff }} L_{i} \cong 0.1077$. Under such conditions, (2) is approximately satisfied. Substituting these values into (1), we get $\eta \cong 100 \%$. This means the studied RCE photodiode structure has been optimized and the resonant wavelength is $1.3 \mu \mathrm{m}$.

To obtain high quantum efficiency, RCE photodetectors have to be operated at the resonant condition. But even at resonance, one still has the flexibility to change the thickness of individual layers as long as the round trip phase delay is $2 k \pi$. As will be described later, one can vary and therefore optimize the device's speed performance while keeping the devices at resonance with high quantum efficiencies by adjusting the thickness of certain layers. If we vary the ratio $L p / L n$ but keep $L_{p}+L_{n}=2 \lambda$ and all other structure parameters the same, we can obtain the quantum efficiencies for devices with various combinations of $L_{p}$ and $L_{n}$ at the resonant condition. Fig. 3 shows the quantum efficiency for the RCE photodiode versus the ratio $L_{p} / L_{n}$ calculated by the TMM method. It can be seen that $\eta$ is nearly $100 \%$ at $L_{p}\left(L_{n}\right)=3 / 2((1 / 2 \lambda), 5 / 4 \lambda(3 / 4 \lambda), \lambda(\lambda), 3 / 4 \lambda(5 / 4 \lambda)$, and $1 / 2 \lambda(3 / 2 \lambda)$. The quantum efficiency changes periodically whenever $L_{p}$ (or $L_{n}$ ) changes by $1 / 2 \lambda$. Because of the nature of this periodic property, there are only two distinct $L_{p} / L_{n}$ ratios (namely, $L_{p}\left(L_{n}\right)=\lambda(\lambda)$ and $3 / 4 \lambda(5 / 4 \lambda)$ ), which have (nearly) $100 \%$ quantum efficiency but different field distributions in the cavity. Fig. 4 shows the light field intensity as a function of position of these two combinations. For $L_{p}=\lambda, L_{n}=\lambda$, we find the maximum of light intensity occurs near the $\mathrm{p}^{+}$edge of the i region, and the result is shown in Fig. 4(a). On the contrary, for $L_{p}=3 / 4 \lambda, L_{n}=5 / 4 \lambda$, the maximum of the field intensity occurs near the $\mathrm{n}^{+}$edge of the $\mathrm{i}$ region [see Fig. 4(b)]. The two different and nonuniform light intensity distributions have different influence on the speed response of this diode and will be discussed in next section. Also shown in Fig. 4 is the light intensity of a conventional p-i-n photodiode for comparison (dashed line); a significant increase in the light intensity can be clearly seen.

\section{FReQuency ReSPONSE OF RCE PhOtodiode}

From Figs. 3 and 4, we know that at the resonant condition when $L_{p}+L_{n}=2 \lambda$ there are two different light field distributions for two different $L_{p} / L_{n}$ ratios. Since the carrier generation rate is proportional to the light intensity, we can thereby obtain different distributions of photogenerated carriers within the i layer. For convenience, we call the light field profile in Fig. 4(a) "Profile 1" and the light field profile in Fig. 4(b) "Profile 2." Fig. 5 shows the photocarrier distributions for these two different light field profiles. In Fig. 5(a), most of the carriers are generated near the $\mathrm{p}^{+}$ edge of the i layer, while in Fig. 5(b) most of the carriers are generated near the $\mathrm{n}^{+}$edge of the $\mathrm{i}$ layer. Taking into account the difference in the drift velocities of the carriers and the nonuniform distribution of the generated carriers in the i region, we calculate the frequency response of the diode below.

$$
\eta=\left[\frac{1+R_{2} \exp \left(-\alpha_{\mathrm{eff}} L_{i}\right)}{1-2 \sqrt{R_{1} R_{2}} \exp \left(-\alpha_{\mathrm{eff}} L_{i}\right) \cos \left(\theta_{k}\right)+R_{1} R_{2} \exp \left(-2 \alpha_{\mathrm{eff}} L_{i}\right)}\right]\left(1-R_{1}\right)\left(1-\exp \left(\alpha_{\mathrm{eff}} L_{i}\right)\right)
$$



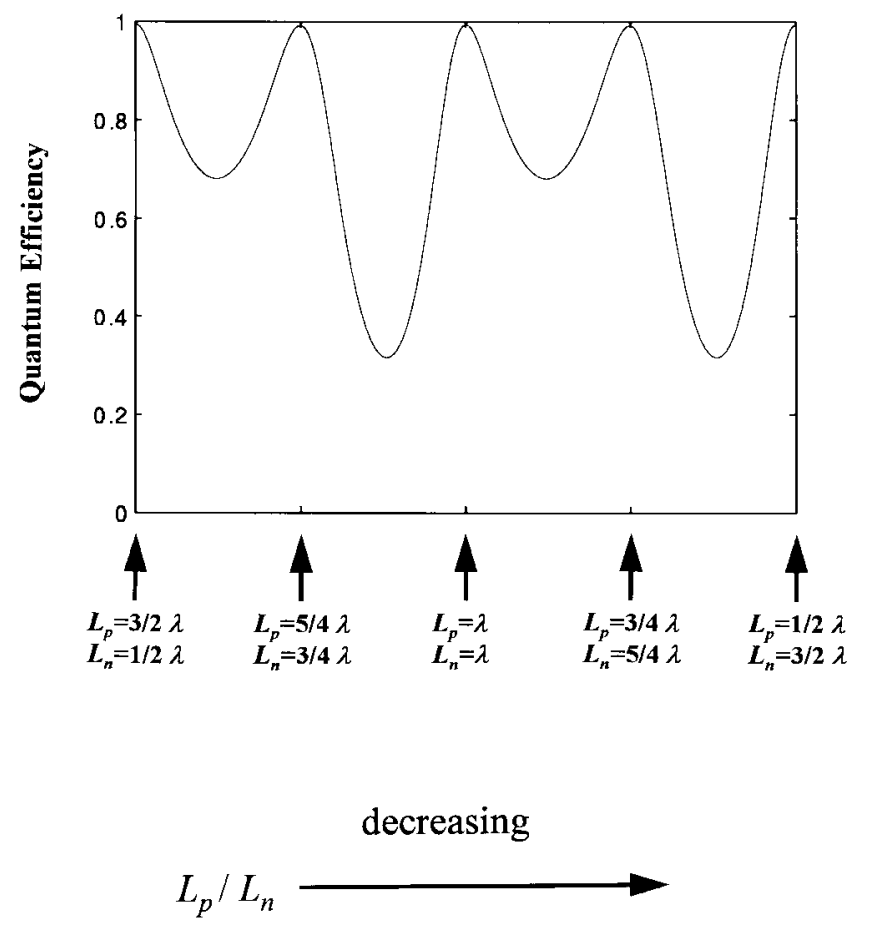

Fig. 3. Thickness dependence of the quantum efficiency for various combinations of $L_{p}$ and $L_{n}$. To operate the RCE photodiode at the resonant condition, $L_{p}+L_{n}$ is kept as a constant of $2 \lambda$.

We start with the linearized continuity equations for the electrons and holes [13]

$$
\begin{aligned}
& \frac{\partial p(x, t)}{\partial t}+v_{p} \frac{\partial p(x, t)}{\partial x}=g(x, t) \\
& \frac{\partial n(x, t)}{\partial t}-v_{n} \frac{\partial n(x, t)}{\partial x}=g(x, t)
\end{aligned}
$$

where $\mathrm{p}$ and $\mathrm{n}$ are the densities of the holes and electrons, $g(x, t)$ is the volume generation rate, and $v_{p}$ and $v_{n}$ are the hole and electron velocities, respectively. We assume a saturated hole velocity of $4.8 \times 10^{6} \mathrm{~cm} / \mathrm{s}$ and a saturated electron velocity of $6.5 \times 10^{6} \mathrm{~cm} / \mathrm{s}$ for the intrinsic layer [10]. At the resonant condition, the light intensity distribution within the $i$ region can be expressed as a function of $\sin ^{2}\left(a_{1} x+a_{2}\right)$ (see Fig. 4). In the present calculations, $a_{1}=2 \pi / 4 L_{i}$, and $a_{2}=0 \sim \pi / 2\left(a_{2}=\pi / 2\right.$ for Profile 1 and $a_{2}=0$ for Profile 2). Consider a sinusoidal modulation of optical power illuminate upon the RCE photodiode, the time variation of the generation rate can be expressed in the following form:

$$
g(x, t)=g_{0} \sin ^{2}\left(a_{1} x+a_{2}\right)(1+m \exp (i \omega t))
$$

where $m$ is a modulation index and $g_{0}$ is a normalization constant. Substituting (4) into (3) together with the boundary conditions $p\left(L_{i}\right)=0$ and $n(0)=0$, we get

$$
\begin{aligned}
& p(x, t)=B_{p}(x)+D_{p}(x, \omega) \exp (i \omega t) \\
& n(x, t)=B_{n}(x)+D_{n}(x, \omega) \exp (i \omega t)
\end{aligned}
$$

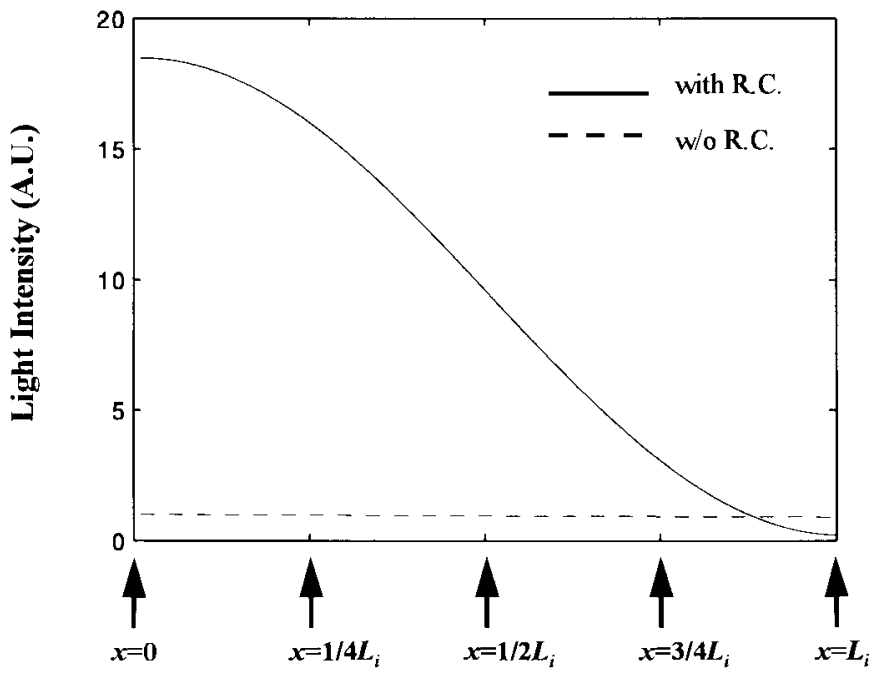

Distance

(a)

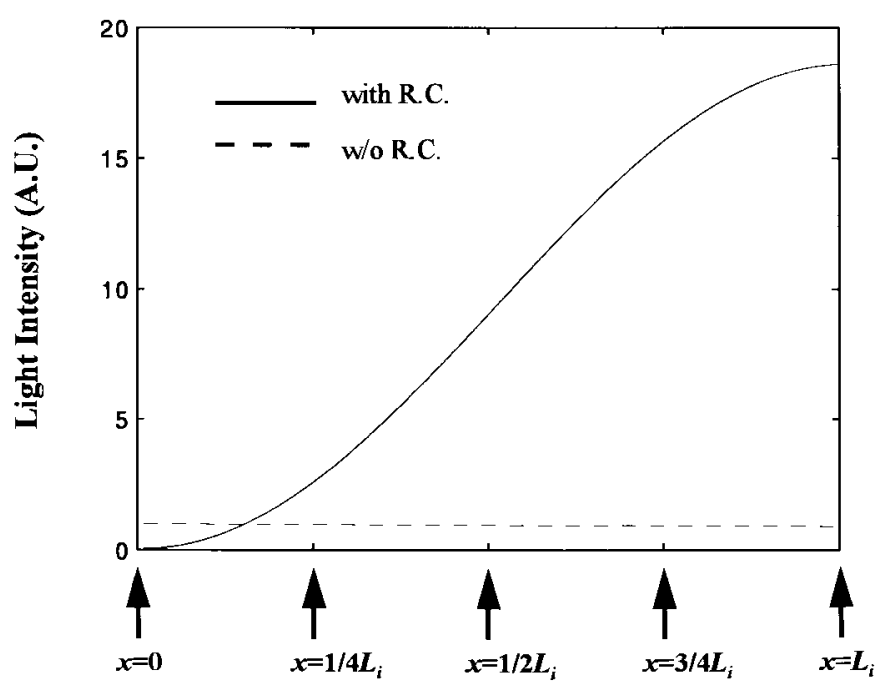

Distance

(b)

Fig. 4. Light field distribution within the $\mathrm{i}$ region of the studied RCE photodiode as a function of position for (a) $L_{p}=\lambda, L_{n}=\lambda$ and (b) $L_{p}=3 / 4 \lambda, L_{n}=5 / 4 \lambda$. The dashed curve is for the conventional photodiode without resonant cavity.

where

$$
\begin{aligned}
B_{p}(x)= & \frac{g_{0}}{4 a_{1} v_{p}}\left\{2 a_{1}\left(x-L_{i}\right)+\sin \left(2 a_{2}\right)\right. \\
& \left.-\sin \left[2 \alpha_{1}\left(x-L_{i}\right)+2 a_{2}\right]\right\} \\
B_{n}(x)= & -\frac{g_{0}}{4 a_{1} v_{n}}\left\{2 a_{1} x+\sin \left(2 \alpha_{2}\right)-\sin \left[2 a_{1} x+2 a_{2}\right]\right\} \\
D_{p}(x, \omega)= & A_{1}\left[A_{2}+A_{3}+A_{4} \cos \left(2 a_{1} x+2 a_{2}\right)\right. \\
& \left.+A_{5} \sin \left(2 a_{1} x+2 a_{2}\right)\right]+C_{1} \exp \left(-i \omega \tau_{p}\right) \\
D_{n}(x, \omega)= & A_{6}\left[A_{7}+A_{8}+A_{9} \cos \left(2 a_{2} x+2 a_{2}\right)\right. \\
& \left.+A_{10} \sin \left(2 a_{1} x+2 a_{2}\right)\right]+C_{2} \exp \left(i \omega \tau_{n}\right) . \quad(6 \mathrm{~d})
\end{aligned}
$$




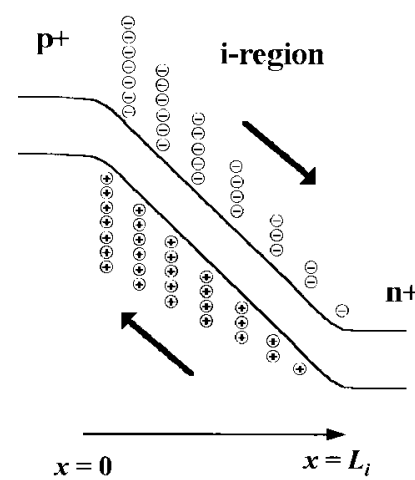

(a)

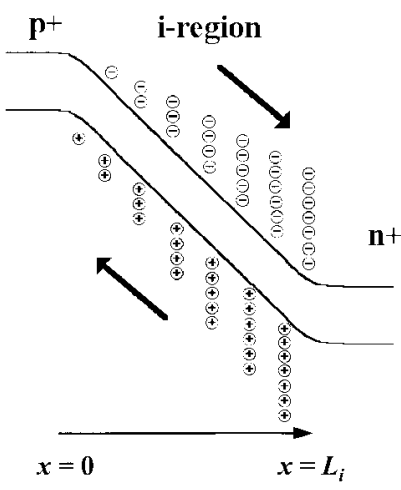

(b)

Fig. 5. Photocarrier distribution profiles for different combinations of $L_{p}$ and $L_{n}$. (a) When $L_{p}=\lambda, L_{n}=\lambda$, the maximum occurs at the $\mathrm{p}^{+}$edge of the i region. (b) When $L_{p}=3 / 4 \lambda, L_{n}=5 / 4 \lambda$, the maximum occurs at the $\mathrm{n}^{+}$edge of the $\mathrm{i}$ region.

$\tau_{p}\left(=L_{i} / v_{p}\right)$ and $\tau_{n}\left(=L_{i} / v_{n}\right)$ are the electron and the hole transit times, respectively, $A_{1} \sim A_{10}$ and $C_{1}, C_{2}$ are given in Appendix A. The current density in the diode is

$$
\begin{aligned}
J(\omega)= & \frac{q}{L_{i}} \int_{0}^{L_{i}}\left[v_{p} p(x, t)+v_{n} n(x, t)\right] d x \\
= & \frac{q}{L_{i}} \int_{0}^{L_{i}}\left[v_{p} B_{p}(x)+v_{n} B_{n}(x)\right] d x \\
& +\frac{q}{L_{i}} \int_{0}^{L_{i}}\left[v_{p} D_{p}(x, \omega)+v_{n} D_{n}(x, \omega)\right] \exp (i \omega t) d x \\
= & J_{0}+J_{s}(\omega) \exp (i \omega t)
\end{aligned}
$$

where $q$ is the electronic charge, $J_{0}$ is the DC component, $J_{s}(\omega)$ is the signal current density, and

$$
J_{s}(\omega)=J_{p}(\omega)+J_{n}(\omega)
$$

where

$$
\begin{aligned}
& J_{p}(\omega)=\frac{q A_{1}}{\tau_{p}}\left\{L_{i}\left(A_{2}+A_{3}\right)+\frac{A_{4}}{2 a_{1}}\left[-\sin \left(2 a_{2}\right)\right.\right. \\
&\left.+\sin \left(2 a_{1} L_{i}+2 a_{2}\right)\right]+\frac{A_{5}}{2 a_{1}}\left[\cos \left(2 a_{2}\right)\right. \\
&\left.-\cos \left(2 a_{1} L_{i}+2 a_{2}\right]\right\}+i C_{1} \frac{q v_{p} \tau_{p}}{\omega} \\
& \cdot\left[-1+\exp \left(-i \omega \tau_{p}\right]\right.
\end{aligned}
$$

$$
\begin{aligned}
J_{n}(\omega)=\frac{q A_{6}}{\tau_{n}}\left\{L_{i}\left(A_{7}+A_{8}\right)+\frac{A_{9}}{2 a_{1}}\left[-\sin \left(2 a_{2}\right)\right.\right. \\
\left.+\sin \left(2 a_{1} L_{i}+2 a_{2}\right)\right]+\frac{A_{10}}{2 a_{1}}\left[\cos \left(2 a_{2}\right)\right. \\
\left.\quad-\cos \left(2 a_{1} L_{i}+2 a_{2}\right]\right\}+i C_{2} \frac{q v_{n} \tau_{n}}{\omega} \\
\cdot\left[1-\exp \left(i \omega \tau_{n}\right)\right] .
\end{aligned}
$$

The frequency response $F\left(\omega, v_{p}, v_{n}, a_{1}, a_{2}\right)$ is given by

$$
F\left(\omega, v_{p}, v_{n}, a_{q}, a_{2}\right)=20 \log \left|\frac{J_{s}(\omega)}{J_{s}(0)}\right|
$$

If the RC effect is included, then

$$
F\left(\omega, v_{p}, v_{n}, a_{1}, a_{2}\right)=20 \log \left\{\frac{\left|\frac{J_{s}(\omega)}{J_{s}(0)}\right|}{\sqrt{1+\omega^{2} R^{2} C^{2}}}\right\}
$$

where $R$ is the sum of the load resistance $\left(R_{L}\right)$ and the diode series resistance $\left(R_{S}\right)$ and $C$ is the diode capacitance plus other parasitic capacitances. In this calculations, $R$ is assumed to be $50 \Omega$ and the diode area is assumed to be $2 \mu \mathrm{m} \times 2 \mu \mathrm{m}$. A reasonable value of $C$ is taken to be $10 \mathrm{fF}$. Fig. 6 plots the frequency response calculated from (9b). If the light field distribution is Profile 2 [Fig. 4(a)], the calculated 3-dB frequency is $204 \mathrm{GHz}$. For Profile 1 [Fig. 4(b)], the calculated 3-dB frequency is about $234 \mathrm{GHz}$, and an increase of $30 \mathrm{GHz}$ is observed. In Profile 1, most of the electronhole pairs are generated near the $\mathrm{p}^{+}$edge of the depletion layer. The holes are collected immediately by the $\mathrm{p}^{+}$layer while the electrons drift through the depletion layer for a time $\tau_{n}\left(=L_{i} / v_{n}\right)$, which depends on the electron saturation drift velocity $v_{n}$. If most of the carriers were generated near the $\mathrm{n}^{+}$edge of the depletion layer (Profile 2), the transit time and the current would be determined by the hole saturation drift velocity $v_{p}$. Since $v_{n}\left(=6.5 \times 10^{6} \mathrm{~cm} / \mathrm{s}\right)$ is greater than $v_{p}\left(=4.8 \times 10^{6} \mathrm{~cm} / \mathrm{s}\right)$, a bandwidth increase for Profile 1 is expected. If the ratio of $v_{n} / v_{p}$ is larger, the difference will be even more evident. The frequency response of the photodiode without a resonant cavity is also shown in Fig. 6 (the dashed-dotted curve) for comparison. The calculated 3$\mathrm{dB}$ frequency is about $216 \mathrm{GHz}$ (Appendix B). For such devices, there is no enhancement for the optical field due to resonance and the light is absorbed almost uniformly in the $\mathrm{i}$ region because the $\mathrm{i}$ layer is very thin (less than $0.1 \mu \mathrm{m}$ ); photocarriers are generated everywhere in the depletion region.

Fig. 7 shows the 3-dB frequency (or bandwidth) as a function of the $L_{p} / L_{n}$ ratio. $L_{p}+L_{n}$ was kept at $2 \lambda$ to ensure the resonant condition. From Figs. 3 and 7, we obtain the gain-bandwidth product which is shown in Fig. 8. We can see that $L_{p}=\lambda, L_{n}=\lambda$ (or thicknesses differ by $1 / 2 \lambda$ ) gives the best gain-bandwidth product and is the optimum design for the present structure. To emphasize further the influence of optical field distribution on the speed of a RCE photodiode, the 


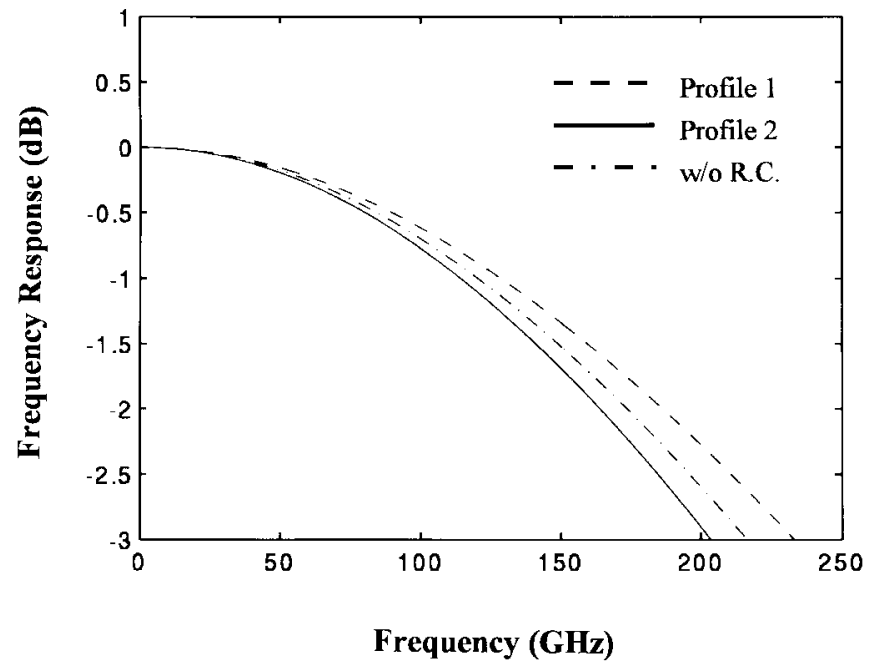

Fig. 6. Calculated frequency response of the studied RCE photodiode. The dashed line shows the response with Profile 1 and the solid line shows the response with Profile 2. Also shown is the calculated response for the conventional photodiode without resonant cavity (dashed-dotted line).

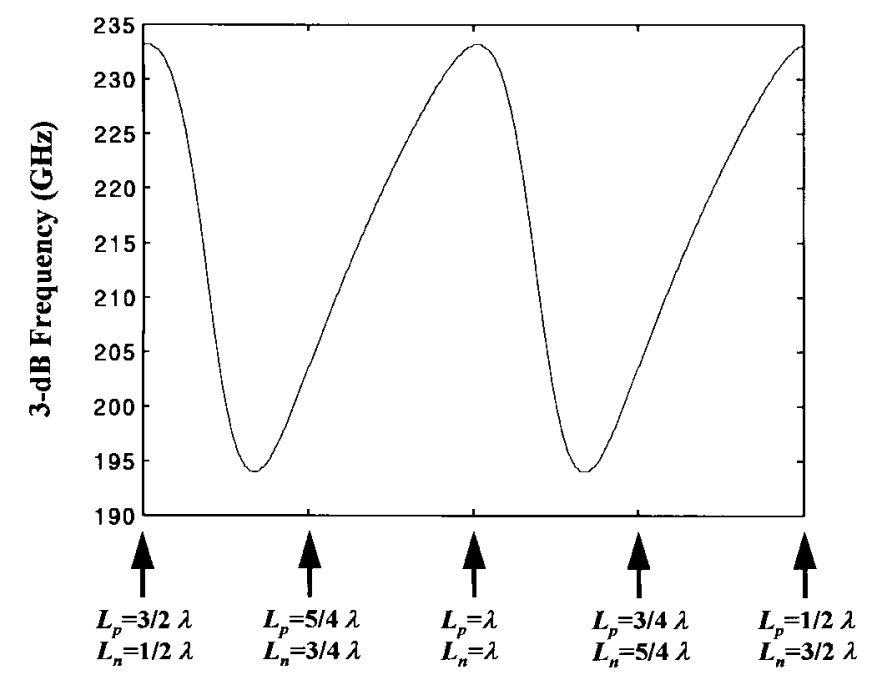

decreasing

$L_{p} / L_{n}$

Fig. 7. Thickness dependence of the 3-dB frequency (or bandwidth) for various combinations of $L_{p}$ and $L_{n}$. To operate the RCE photodiode at the resonant condition, $L_{p}+L_{n}$ is kept as a constant of $2 \lambda$.

response of the photodiode to an impulse of light is computed in the next section.

\section{IMPUlSe Response AND Step Response}

\section{A. Impulse Response}

Consider a situation in which an impulse of optical power generates $N$ electron-hole pairs at $t=0$ according to the light field distribution. As described earlier, the carrier density

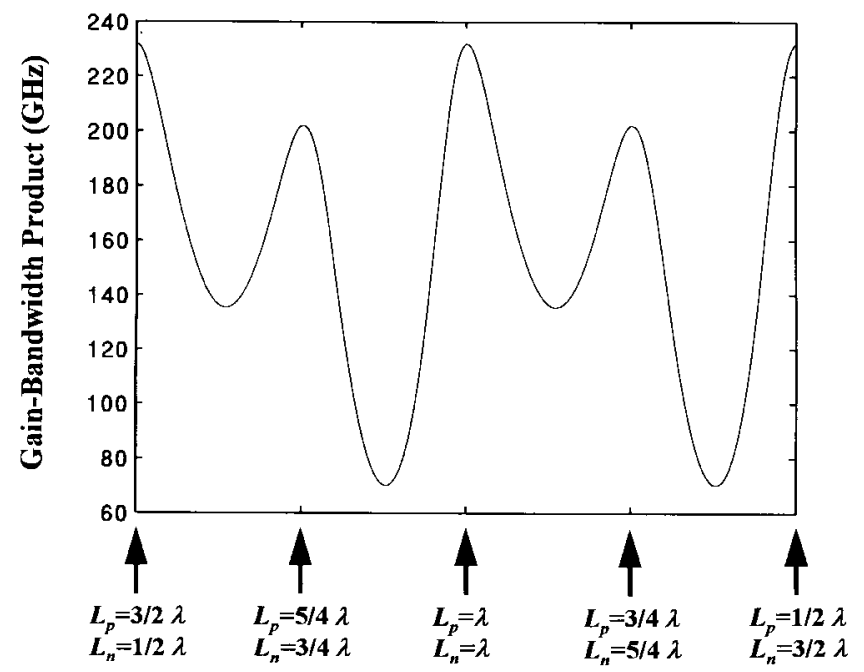

\section{decreasing}

$$
L_{p} / L_{n}
$$

Fig. 8. Thickness dependence of the gain-bandwidth product for various combinations of $L_{p}$ and $L_{n}$. The maximum is about $231 \mathrm{GHz}$ at $L_{p}=\lambda, L_{n}=\lambda$ (Profile 1$)$.

distribution function $n(x)$ can be expressed as

$$
n(x)=N_{0} \sin ^{2}\left(a_{1} x+a_{2}\right)
$$

where $N_{0}$ is a normalization factor and

$$
\begin{aligned}
N & =\int_{0}^{L_{i}} n(x) d x \\
& =\int_{0}^{L_{i}} N_{0}\left[\sin ^{2}\left(a_{1} x+a_{2}\right)\right] d x
\end{aligned}
$$

from which we get

$$
N_{0}=\frac{4 a_{1} N}{\sin \left(2 a_{2}\right)+2 a_{1} L_{i}-\sin \left(2 a_{1} L_{i}+2 a_{2}\right)} .
$$

After a period of time $t$, part of the carriers drift out of the depletion region, and the average electron density left within the i layer becomes

$$
\begin{aligned}
n(t)= & \frac{1}{L_{i}}\left\{\int_{0}^{L_{i}} N_{0} \sin ^{2}\left[a_{1}\left(x-v_{n} t\right)+a_{2}\right] d x\right\} \\
& \cdot \mathrm{U}\left(\tau_{n}-t\right), \quad t \geq 0 \\
= & \frac{N_{0}}{L_{i}}\left\{\frac{1}{2}\left(L_{i}-v_{n} t\right)+\frac{1}{4 a_{1}}\left[\sin \left(2 a_{2}\right)\right.\right. \\
& \left.\left.-\sin \left(2 a_{1}\left(L_{1}-v_{n} t\right)+2 a_{2}\right)\right]\right\} \mathrm{U}\left(\tau_{n}-t\right), \\
& t \geq 0
\end{aligned}
$$


where $U$ is the unit step function. The electron current density is

$$
\begin{aligned}
J_{n}(t)= & q n(t) v_{n} \\
= & \frac{q N_{0}}{4 a_{1} \tau_{n}}\left\{2 a_{1}\left(L_{i}-v_{n} t\right)+\sin \left(2 a_{2}\right)\right. \\
& \left.\quad-\sin \left[2 a_{1}\left(L_{i}-v_{n} t\right)+2 a_{2}\right]\right\} \\
& \cdot \mathrm{U}\left(\tau_{n}-t\right), \quad t \geq 0 .
\end{aligned}
$$

Similarly, the number of holes inside the i layer at time $t$ is

$$
\begin{aligned}
p(t)= & \frac{1}{L_{i}}\left\{\int_{0}^{L-i-v_{p} t} N_{0} \sin ^{2}\left[a_{1}\left(x+v_{p} t\right)+a_{2}\right] d x\right\} \\
& \cdot \mathrm{U}\left(\tau_{p}-t\right), \quad t \geq 0 \\
= & \frac{N_{0}}{L_{i}}\left\{\frac{1}{2}\left(L_{i}-v_{p} t\right)+\frac{1}{4 a_{1}}\left[\sin \left(2 a_{1} v_{p} t+2 a_{2}\right)\right.\right. \\
& \left.\left.\quad-\sin \left(2 a_{1} L_{i}+2 a_{2}\right)\right]\right\} \mathrm{U}\left(\tau_{p}-t\right), \quad t \geq 0
\end{aligned}
$$

and the hole current density is

$$
\begin{aligned}
J_{p}(t)= & q p(t) v_{p} \\
=\frac{q N_{0}}{4 a_{1} \tau_{p}} & \left\{2 a_{1}\left(L_{i}-v_{p} t\right)+\sin \left(2 a_{1} v_{p} t+2 a_{2}\right)\right. \\
& \left.\quad-\sin \left(2 a_{1} L_{i}+2 a_{2}\right)\right\} \mathrm{U}\left(\tau_{p}-t\right), \quad t \geq 0 .
\end{aligned}
$$

The total current density is then

$$
J_{\mathrm{imp}}(t)=J_{n}(t)+J_{p}(t)
$$

Fig. 9 shows the calculated current impulse response. An important figure of merit for digital circuit applications is the fall time, $t_{f}$, which is defined as the time when the current density falls from $90 \%$ of the initial value to $10 \%$ of the initial value. The calculate $t_{f}$ is $1.570 \mathrm{ps}$ for Profile 2 and $1.086 \mathrm{ps}$ for Profile 1. A 50\% improvement in $t_{f}$ is obtained. Since most of the holes in Profile 2 have to take a longer time to drift out of the i region, a clear "tail" is observed in Fig. 9, and that explains why Profile 2 has a much longer $t_{f}$. So, for the purpose of high-bit-rate communication, it is a better choice to use Profile 1 in the design of a RCE photodetector. Also shown in Fig. 9 is the impulse response for the photodiode without a resonant cavity; the calculated $t_{f}$ is $1.289 \mathrm{ps}$ (see Appendix C).

\section{B. Step Response}

Since the continuity equations in (3) are linear, the response to an arbitrary input function $X(t)$ is then found by convolving impulse response, $J_{\mathrm{imp}}$, with $X(t)$ [14]:

$$
J(t)=J_{\mathrm{imp}} * X(t)=\int_{-\infty}^{\infty} J_{\mathrm{imp}}\left(t^{\prime}\right) X\left(t-t^{\prime}\right) d t^{\prime}
$$

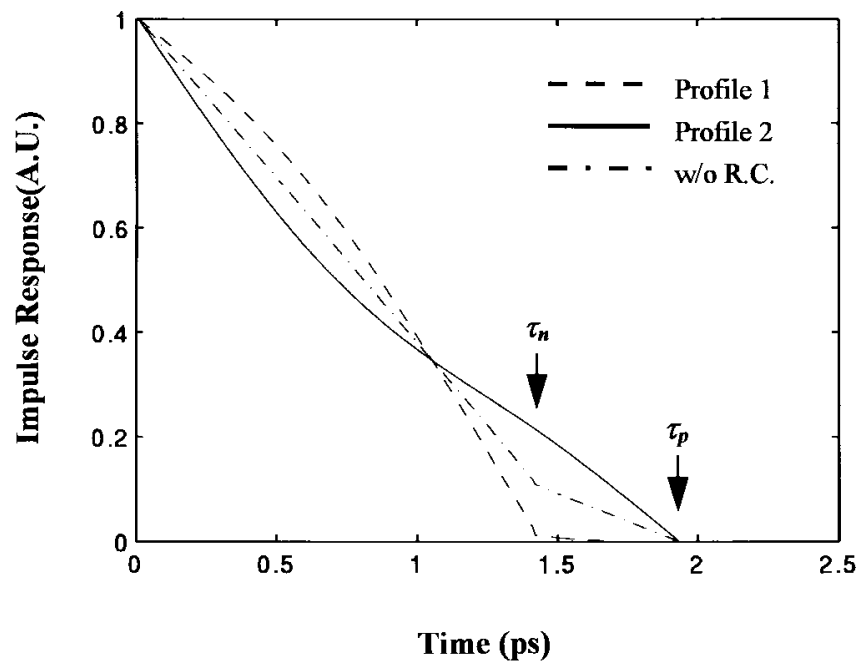

Fig. 9. Impulse response for the studied RCE photodiode. The dashed line shows the response with Profile 1 and the solid line shows the response with Profile 2. Also shown is the calculated response for the conventional photodiode without resonant cavity (dashed-dotted line).

For unit step response,

$$
X(t)= \begin{cases}0, & t<0 \\ U(t), & t>0\end{cases}
$$

Substituting (13) and (15) into (14), we have

$$
J_{\text {step }}(t)=J_{n, \text { step }}(t)+J_{p, \text { step }}(t)
$$

where

$$
\begin{aligned}
& J_{n, \text { step }}(t)=\frac{q N_{0}}{8 a_{1}^{2} L_{i}}\left\{\cos \left(2 a_{1} L_{i}+2 a_{2}\right)+4 a_{1}^{2} L_{i} v_{n} t\right. \\
& -2 a_{1}^{2} v_{n}^{2} t^{2}-\cos \left[2 a_{1}\left(L_{i}-v_{n} t\right)+2 a_{2}\right] \\
& \left.+2 a_{1} v_{n} \sin \left(2 a_{2}\right) t\right\}, \quad 0 \leq t<\tau_{n} \\
& =\frac{q N_{0}}{8 a_{1}^{2} L_{i}}\left\{\cos \left(2 a_{1} L_{i}+2 a_{2}\right)+2 a_{1}^{2} L_{i}^{2}\right. \\
& \left.-\cos \left(2 a_{2}\right)+2 a_{1} L_{i} \sin \left(2 a_{2}\right)\right\}, \quad t \geq \tau_{n} . \\
& J_{p, \text { step }}(t)=\frac{q N_{0}}{8 a_{1}^{2} L_{i}}\left\{\cos \left(2 a_{2}\right)+4 a_{1}^{2} L_{i} v_{p} t-2 a_{1}^{2} v_{p}^{2} t^{2}\right. \\
& -\cos \left(2 a_{1} v_{p} t+2 a_{2}\right) \\
& \left.-2 a_{1} v_{p} \sin \left(2 a_{1} L_{i}+2 a_{2}\right) t\right\}, \quad 0 \leq t<\tau_{p} \\
& =\frac{q N_{0}}{8 a_{1}^{2} L_{i}}\left\{\cos \left(2 a_{2}\right)+2 a_{1}^{2} L_{i}^{2}-\cos \left(2 a_{1} L_{i}+2 a_{2}\right)\right. \\
& \left.-2 a_{1} L_{i} \sin \left(2 a_{1} L_{i}+2 a_{2}\right)\right\}, \quad t \geq \tau_{p} .
\end{aligned}
$$

The calculated step response of (16) is plotted in Fig. 10. If we define the rise time $t_{r}$ as the time when $J_{\text {step }}$ changes from $10 \%$ of the final value to $90 \%$ of the final value, the calculated $t_{r}$ is $0.945 \mathrm{ps}$ for Profile 1, $1.234 \mathrm{ps}$ for Profile 2, and $1.078 \mathrm{ps}$ for the conventional photodiode (see Appendix D), respectively. The influence of the light field distribution on the performance of step response is again very clear. 


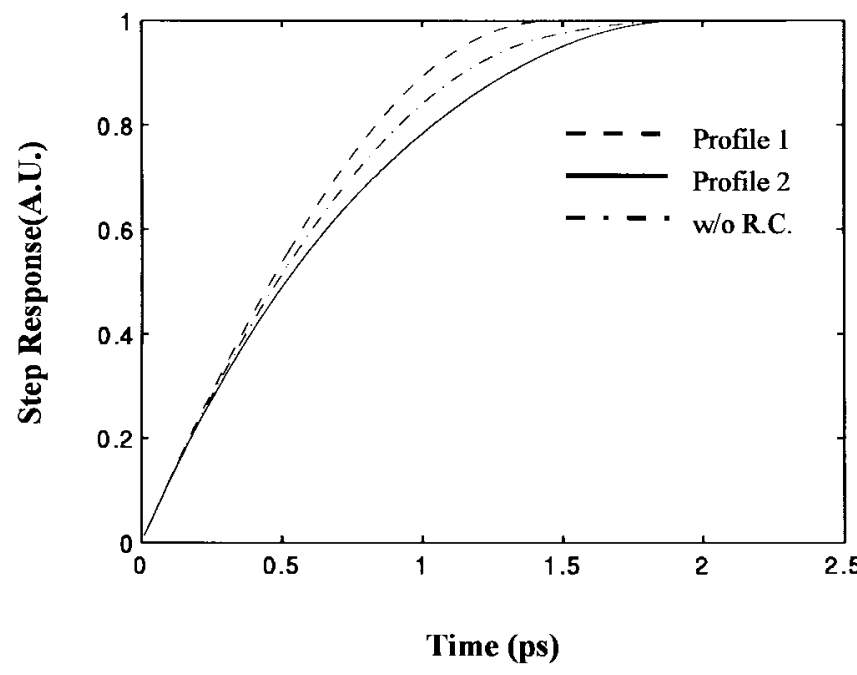

Fig. 10. Step response for the studied RCE photodiode. The dashed line shows the response with Profile 1 and the solid line shows the response with Profile 2. Also shown is the calculated response for the conventional photodiode without resonant cavity (dashed-dotted line).

\section{CONCLUSION}

We have studied the high-speed performance of a doubleheterostructure RCE p-i-n photodiode. At the resonant condition, by varying the length of $L_{p}\left(L_{n}\right)$ while keeping $L_{p}+L_{n}$ constant, we can obtain different light field distributions. By properly choosing $L_{p}\left(L_{n}\right)$, we can obtain an optimum structure where the maximum light intensity occurs near the $\mathrm{p}^{+}$edge of the $\mathrm{i}$ layer. In such a structure, most of the generated holes are collected immediately by the $\mathrm{p}^{+}$layer while the electrons drift through the depletion layer with a larger drift velocity. By theoretically solving the linearized continuity equations, the calculated $3-\mathrm{dB}$ frequency, gainbandwidth product, impulse response, and step response all demonstrate the superiority of Profile 1 over Profile 2. So in a RCE structure, one can take advantage of the flexibility of cavity design to tailor the light field distribution in the absorption region to improve the device speed. This concept, demonstrated in a photodetector in this work, should also be useful for other optoelectronic devices.

\section{APPENDIX A}

The parameters used in (6) are

$$
\begin{aligned}
& A_{1}=\frac{i g_{0} m}{2 \omega\left(\omega^{2}-4 a_{1}^{2} v_{p}\right)} \\
& A_{2}=4 a_{1}^{2} v_{p}^{2} \\
& A_{3}=-\omega^{2} \\
& A_{4}=\omega^{2}
\end{aligned}
$$

$$
\begin{aligned}
A_{5} & =-2 i a_{1} v_{p} \omega \\
A_{6} & =\frac{i g_{0} m}{2 \omega\left(\omega^{2}-4 a_{1}^{2} v_{n}\right)} \\
A_{7} & =4 a_{1}^{2} v_{n}^{2} \\
A_{8} & =A_{3} \\
A_{9} & =A_{4} \\
A_{10} & =2 i a_{1} v_{p} \omega \\
C_{1} & =-A_{1}\left[A_{2}+A_{3}+A_{4} \cos \left(2 a_{1} L_{i}+2 a_{2}\right)\right. \\
& \left.\quad+A_{5} \sin \left[2 a_{1} L_{i}+2 a_{2}\right)\right] \exp \left(i \omega \tau_{p}\right) \\
C_{2}= & -A_{6}\left[A_{7}+A_{8}+A_{9} \cos \left(2 a_{2}\right)+A_{10} \sin \left(2 a_{2}\right)\right] .
\end{aligned}
$$

\section{APPENDIX B}

The frequency response of a conventional $\mathrm{p}-\mathrm{i}-\mathrm{n}$ photodetector with light incident from the p side is (B1) [15], shown at the bottom of the page. For n-side illumination, (B1) is valid if the subscripts $\mathrm{p}$ and $\mathrm{n}$ are switched.

\section{APPENDIX C}

For a p-side illuminated $\mathrm{p}-\mathrm{i}-\mathrm{n}$ photodetector without a resonant cavity, we have

$$
\begin{aligned}
N & =\int_{0}^{L_{i}} N_{0} \exp (-\alpha x) d x \\
& =\frac{N_{0}}{\alpha}\left[1-\exp \left(-\alpha L_{i}\right)\right] .
\end{aligned}
$$

From (C1), we get

$$
N_{0}=\frac{\alpha N}{1-\exp \left(-\alpha L_{i}\right)} .
$$

The average electron density within the $\mathrm{i}$ layer is

$$
\begin{aligned}
n(t) & =\frac{1}{L_{i}}\left\{\int_{v_{n} t}^{L_{i}} N_{0} \exp \left[-\alpha\left(x-v_{n} t\right)\right] d x\right\} \mathrm{U}\left(\tau_{n}-t\right) \\
& =\frac{N_{0}}{\alpha L_{i}}\left\{1-\exp \left[-\alpha\left(L_{i}-v_{n} t\right)\right]\right\} \mathrm{U}\left(\tau_{n}-t\right)
\end{aligned}
$$

and the electron current density is

$$
\begin{aligned}
J_{n}(t)= & q n(t) v_{n} \\
= & \frac{q N_{0}}{\alpha \tau_{n}}\left\{1-\exp \left[-\alpha\left(L_{i}-v_{n} t\right)\right]\right\} U\left(\tau_{n}-t\right), \\
& \quad t \geq 0 .
\end{aligned}
$$

Similarly,

$$
\begin{aligned}
p(t) & =\frac{1}{L_{i}}\left\{\int_{0}^{L_{i}-v_{p} t} N_{0} \exp \left[-\alpha\left(x+v_{n} t\right)\right] d x\right\} \mathrm{U}\left(\tau_{p}-t\right) \\
& =\frac{N_{0}}{\alpha L_{i}}\left\{-\exp \left(-\alpha L_{i}\right)+\exp \left(-\alpha \nu_{p} t\right)\right\} \mathrm{U}\left(\tau_{p}-t\right)
\end{aligned}
$$

$$
\begin{aligned}
\frac{J(\omega)}{J(0)}=\frac{1}{1-\exp \left(-\alpha L_{i}\right)}[ & \frac{\exp \left(i \omega \tau_{p}-\alpha L_{i}\right)-1}{i \omega \tau_{p}-\alpha L_{i}}-\exp \left(-\alpha L_{i}\right) \frac{\exp \left(i \omega \tau_{p}\right)-1}{i \omega \tau_{p}} \\
& \left.+\frac{\exp \left(i \omega \tau_{n}\right)-1}{i \omega \tau_{n}}+\exp \left(-\alpha L_{i}\right) \frac{1-\exp \left(\alpha L_{i}+i \omega \tau_{n}\right)}{\alpha L_{i}+i \omega \tau_{n}}\right]
\end{aligned}
$$


and the hole current density is

$$
\begin{aligned}
J_{p}(t)= & q p(t) v_{p} \\
= & \frac{q N_{0}}{\alpha \tau_{p}}\left\{-\exp \left(-\alpha L_{i}\right)+\exp \left(-\alpha v_{p} p t\right)\right\} U\left(\tau_{p}-t\right), \\
& \quad t \geq 0 .
\end{aligned}
$$

The total current density is

$$
J_{\mathrm{imp}}(t)=J_{n}(t)+J_{p}(t) .
$$

\section{APPENDIX D}

The step response for a conventional $\mathrm{p}-\mathrm{i}-\mathrm{n}$ photodetector with p-side illumination can be calculated directly by convolving the impulse response (C2) with unit step function $X(t)$. Substituting (C2), $X(t)$ into (14), we obtain

$$
J_{\text {step }}(t)=J_{n, \text { step }}(t)+J_{p, \text { step }}(t)
$$

where

$$
\begin{aligned}
& J_{n, \text { step }}(t)= \frac{q N_{0}}{\alpha \tau_{n}}\left\{t+\frac{1}{\alpha v_{n}} \exp \left(-\alpha L_{i}\right)\left[1-\exp \left(\alpha v_{n} t\right)\right]\right\}, \\
& \quad 0 \leq t<\tau_{n} \\
&=\frac{q N_{0}}{\alpha \tau_{n}}\left\{\tau_{n}-\frac{1}{\alpha v_{n}}\left[1-\exp \left(-\alpha L_{i}\right)\right]\right\} \\
& \quad t \geq \tau_{n}
\end{aligned}
$$

and

$$
\begin{aligned}
J_{p, \text { step }}(t)= & \frac{q N_{0}}{\alpha \tau_{p}}\left\{-t \exp \left(-\alpha L_{i}\right)+\frac{1}{\alpha v_{p}}\left[1-\exp \left(\alpha v_{p} t\right)\right]\right\}, \\
& 0 \leq t<\tau_{p} \\
= & \frac{q N_{0}}{\alpha \tau_{p}}\left\{\frac{1}{\alpha v_{p}}\left(\tau_{p}-\frac{1}{\alpha v_{p}}\right) \exp \left(-\alpha L_{i}\right)\right\} \\
& t \geq \tau_{p} .
\end{aligned}
$$

\section{REFERENCES}

[1] H. David Law, Semiconductor and Semimetals, Lightwave Communication Technology, Part D, vol. 22, p. 454, 1985

[2] A. L. Gutierrez-Aitken, K. Yang, G. I. Haddad, P. Bhattacharya, and M. Lunardi, "16-Ghz bandwidth InAlAs-InGaAs monolithically integrated p-i-n/HBT photoreceiver," IEEE Photon. Technol. Lett., vol. 7, pp. 1339-1341, 1995.
[3] K. Kishino, M. S. Unlu, J. I. Chyi, J. Reed, L. Arsenault, and H. Morkoc, "Resonant cavity-enhanced (RCE) photodetectors," IEEE J. Quantum Electron., vol. 27, pp. 2025-2034, 1991.

[4] A. Salvador, F. Huang, B. Sverdlov, A. E. Botchkarev, and H. Morkoc, "InP/InGaAs resonant cavity enhanced photodetector and light emitting diode with external mirrors on Si," Electron. Lett., vol. 30, pp. 1527-1529, 1994.

[5] P. Guy, K. Woodbridge, S. K. Haywood, and M. Hopkinson, "Highly doped $1.55 \mu \mathrm{m} \mathrm{Ga}_{x} \mathrm{In}_{1-x}$ As/InP distributed Bragg reflector stacks," Electron. Lett., vol. 30, pp. 1526-1527, 1994

[6] C. C. Barron, C. J. Mahon, B. J. Thibeault, G. Wang, W. Jiang, L. A. Goldren, and J. E. Bowers, "Resonant-cavity-enhanced pin photodetector with $17 \mathrm{GHz}$ bandwidth-efficiency product," Electron. Lett., vol. 30, pp. 1796-1797, 1994.

[7] I. H. Tan, E. L. Hu, J. E. Bowers, and B. I. Miller, "Modeling and performance of wafer-fused resonant-cavity enhanced photodetectors," IEEE Quantum Electron., vol. 31, pp. 1863-1875, 1995

[8] Y. G. Wey, D. L. Crawford, K. Giboney, J. E. Bowers, and M. J. Rodwell, "Ultrafast graded double-heterostructure GaInAs/InP photodiode," Appl. Phys. Lett., vol. 58, pp. 2156-2158, 1991.

[9] Y. G. Wey, K. S. Giboney, J. E. Bowers, M. J. W. Rodwell, P. Silvestre, P. Thiagarajan, and G. Y. Robinson, "108 Ghz GaInAs/InP p-i-n photodiodes with integrated bias tees and matched resistors," IEEE Photon. Technol. Lett., vol. 5, pp. 1310-1312, 1993.

[10] J. E. Bowers and C. A. Burrus, "Ultrawide-band long-wavelength p-i-n photodetectors," J. Lightwave Technol., vol. LT-5, pp. 1339-1350, 1987.

[11] G. R. Fowles, Introduction to Modern Optics. New York: Holt, Rinehart and Winston, 1975, p. 100.

[12] M. S. Unlu, K. Kishino, H. J. Liaw, and H. Markoc, "A theoretical study of resonant cavity-enhanced photodetectors with Ge and $\mathrm{Si}$ active regions," J. Appl. Phys., vol. 71, pp. 4049-4058, 1992.

[13] G. Lucovsky, R. F. Schwarz, and R. B. Emmons, "Transit-time considerations in p-i-n diodes," J. Appl. Phys., vol. 35, pp. 622-628, 1964.

[14] A. B. Carlson, Communication Systems, 2nd ed. New York: McGrawHill, 1975.

[15] J. E. Bowers, C. A. Burrus, and R. J. Mccoy, "InGaAs pin photodetectors with modulation response to millimeter wavelengths," Electron. Lett., vol. 21, pp. 812-814, 1985.

Hsin-Han Tung, photograph and biography not available at the time of publication.

Chien-Ping Lee (M'80-SM'94), photograph and biography not available at the time of publication. 